\title{
s.mentaten

\section{Low serum level of apolipoprotein A1 is an indicator of severity in patients with coronavirus disease 2019}

\section{Yayun Yang}

HwaMei Hospital, University of Chinese Academy of Sciences

\section{Zhe Zhu}

HwaMei Hospital, University of Chinese Academy of Sciences https://orcid.org/0000-0003-3450-7259

\section{Lingyan Fan}

HwaMei Hospital, University of Chinese Academy of Sciences

\section{Shuyuan Ye}

HwaMei Hospital, University of Chinese Academy of Sciences

\section{Kehong Lou}

HwaMei Hospital, University of Chinese Academy of Sciences

\section{Xin Hua}

HwaMei Hospital, University of Chinese Academy of Sciences

\section{Zuoan Huang}

HwaMei Hospital, University of Chinese Academy of Sciences

\section{Qiaoyun Shi}

HwaMei Hospital, University of Chinese Academy of Sinences

Guosheng Gao ( $\nabla 45926922 @ q q . c o m$ )

\section{Research}

Keywords: COVID-19, SARS-CoV-2, blood lipid, ApoA1

Posted Date: May 29th, 2020

DOI: https://doi.org/10.21203/rs.3.rs-31251/v1

License: (c) (i) This work is licensed under a Creative Commons Attribution 4.0 International License.

Read Full License 


\section{Abstract}

Background: Recently, dyslipidemia was observed in the patients of coronavirus disease 2019 (COVID19), especially in the severe cases. This study aimed to explore the predictive value of blood lipid for the severity of COVID-19.

Methods: All patients with COVID-19 who admitted to HwaMei Hospital, University of Chinese Academy of Sciences from January 23 to April 20, 2020 were included in this retrospective study. General clinical characteristics and laboratory data (including blood lipid parameters) were obtained, and their predictive values for the severity were analyzed.

Results: 142 consecutive patients with COVID-19 were included. There were 125 cases in the non-severe group and 17 cases in the severe group. Total cholesterol, high-density lipoprotein cholesterol (HDL-C), low-density lipoprotein cholesterol (LDL-C), apolipoprotein A1 (ApoA1) at baseline were significantly lower in the severe group. ApoA1 and interleukin-6 (IL-6) were recognized as independent risk factors for the severity of COVID-19. ApoA1 had the highest area under the receiver operator characteristic curve (AUROC) among all the single markers (AUROC: 0.896, 95\% Cl: 0.834-0.941). Moreover, the risk model established upon ApoA1 and IL-6 enhanced the predictability (AUROC: 0.977, 95\% Cl: 0.932-0.995). On the other hand, ApoA1 in the severe group elevated during the treatment, there was no significant difference between the severe and non-severe groups in the recovery stage of disease.

Conclusion: The profile of blood lipid in the severe patients with COVID-19 is quite different from that in the non-severe cases. Serum ApoA1 could severe as a good marker to reflect the severity of COVID-19.

\section{Introduction}

The recent emerged pathogenic severe acute respiratory syndrome coronavirus 2 (SARS-CoV-2) is a highly transmissible coronavirus which caused coronavirus disease 2019 (COVID-19) since December 2019 , and spread worldwide rapidly. As of May 13, 2020, SARS-CoV-2 has caused 4170424 confirmed cases and 287399 deaths (https://www.who.int/emergencies/diseases/novel-coronavirus2019/situation-reports). The ongoing COVID-19 pandemic has brought a huge pressure to the medical system globally. Although about $80 \%$ patients infected with SARS-CoV2 are mild [1], the remain severe cases may suffer from acute respiratory distress, multi-organ failure and death [2]. Therefore, it is necessary to discriminate the severe cases from mild cases.

Previous studies have found that the development of severe COVID-19 is associated with age, underlying disease, and severe patients are vulnerable to suffer from aberrant inflammation reaction and cytokine storm $[3,4]$. Consequently, some clinical characteristic, inflammation index and cytokines have been used as markers to reflect the severity of COVID-19 by us and others $[5,6]$. Recently, emerging evidence suggested that lipid metabolism dysregulation may involve into the progression of COVID-19 revealed bymass spectrometry (MS)-based proteomics analysis [7-9]. Although MS analysis is not common 
performed, blood lipid is routinely examined by automatic biochemical instrument in clinically laboratory. Thus, blood lipid maybe considered as a potential and available marker for the severity of COVID-19.

In order to determine the predictive value of blood lipid for the severity of COVID-19, a retrospective study was carried out in Ningbo HwaMei Hospital, University of Chinese Academy of Sciences.

\section{Materials And Methods}

\section{Study site and design}

This was a single-center retrospective study performed at HwaMei Hospital, University of Chinese Academy of Sciences, a 2100-bed tertiary general hospital integrated with medical treatment, health care, disease prevention, teaching and scientific research. It was a designated hospital for COVID-19 during the SARS-CoV-2 epidemic, and received most of the patients with COVID-19 at Ningbo, Zhejiang province, China. This study was approved by the institutional ethics committee (Certificate no. PJ-NBEY-KY-2020061-01).

\section{Patient selection and data collection}

All consecutive patients with confirmed COVID-19 admitted to HwaMei Hospital, University of Chinese Academy of Sciences from January 23 to April 20, 2020 were enrolled. The diagnosis of COVID-19 and its severity were determined according to the Diagnosis and Treatment Protocol for Novel Coronavirus Infection-Induced Pneumonia (Trial Version 6). All patients were divided into mild, moderate, severe and critical based on the severity of COVID-19. Mild and moderate patients were classified into the non-severe group, while severe and critical patients were categorized as the severe group. Severe patients should have one of the following features: a) shortness of breath with respiration rate (RR) more than 30 times per minute; $b$ ) blood oxygen saturation $\leq 93 \%$ at the state of rest; $c$ ) arterial blood oxygen partial pressure / inhaled oxygen concentration $\leq 300 \mathrm{~mm} \mathrm{Hg}$. Lesion progressed more than $50 \%$ within $24-48$ hours on pulmonary imaging were also considered as severe patients. Critical patients meet any of the following: a) respiratory failure, needing mechanical ventilation for therapy; b) shock; c) additional other organ failure, needing treatment in the intensive care unit.

General clinical characteristics including gender, age, comorbidities, initial symptoms and treatment, laboratory examination and follow-up data were collected from the electronic medical record (EMR).

\section{Laboratory examination}

SARS-CoV-2 RNA was detected by the Real-Time Fluorescent reverse transcription polymerase chain reaction (RT-PCR) (Daangene, China). Routine blood test was analyzed with the XS-1000i automatic hematology analyzer (Sysmex, Japan). Blood coagulation test was determined by the ACLTOP750 
coagulation analyzer (Instrumentation Laboratory, USA). Biochemical parameters, including blood lipid, liver function indices and renal function indices, etc. were tested using a fully automatic biochemical analyzer (ADVIA2400, Siemens, Germany). Cytokines were detected by the flow cytometry with human Th1/2 cytokine kit (CellGene, China).

\section{Statistical analysis}

SPSS statistical 16.0 software (IBM, Armonk, NY, USA) and GraphPad PRISM 5.0 software (GraphPad Software, San Diego, CA, USA) were used for statistical analysis. Normally and non-normally distributed continuous data were expressed as mean \pm SD (standard deviation) and medians and interquartile range (IQR), respectively. Categorical variables were descripted as number (\%). We assessed the differences between the non-severe and severe group using Student's $t$-test or Mann-Whitney $U$ test for the normally or non-normally distributed continuous data, and using chi square or Fisher's exact test for the categorical variables. We used multivariate logistic regression analysis to explore independent risk factors for the severity of COVID-19, and performed receiver operator characteristic (ROC) curves to assess their predictive values. Correlations between different variables were determined by Spearman rank correlation analysis. A $P$-value $<0.05$ indicates statistical significance.

\section{Results}

\section{General clinical characteristics}

142 consecutive patients with confirmed COVID-19 were included in this study. The mean age was 49.10 \pm 16.36 years, $55(38.73 \%)$ patients were men. Hypertension $(37,26.06 \%)$, diabetes $(12,8.45 \%)$, hepatic disease $(10,7.04 \%)$ and chronic lung disease $(9,6.34 \%)$ were the most common comorbidities. Fever $(84,59.15 \%)$ was the leading initial symptom, followed by cough $(61,42.96 \%)$, expectoration (32, $22.54 \%$ ) and fatigue $(27,19.01 \%)$. There were still 18 cases $(12.68 \%)$ showed no obvious symptoms when admission. All included patients received antivirals treatment, besides, 88 (61.97\%), 53 (37.32\%) and $52(36.62 \%)$ patients were treated with gamma globulin, oxygen and antibiotics, respectively.

Among the 142 patients, 125 (88.03\%) patients (19 mild and 106 moderate) were classified into the nonsevere group, and 17 (11.97\%) patients (14 severe and 3 critical) were categorized as the severe group. There were significant differences in age $(56.88 \pm 11.61$ years vs $48.04 \pm 16.66$ years, $P=0.010)$, body mass index (BMI) $(26.13 \pm 5.47 \mathrm{~kg} / \mathrm{m} 2$ vs $23.50 \pm 3.42 \mathrm{~kg} / \mathrm{m} 2, \mathrm{P}=0.007)$, hypertension (9 [52.94\%] vs 28 [22.40\%], $P=0.007)$ and fever (14 [82.35\%] vs $70[56.00 \%], P=0.038)$ between the severe and non-severe groups. As for clinical treatment, there were higher proportion in the severe group who received glucocorticoids, antibiotics, oxygen, invasive mechanical ventilation and intensive care unit treatment when compared with the non-severe group (Table 1). 
Table 1

General clinical characteristics of patients with confirmed COVID-19.

\begin{tabular}{|c|c|c|c|c|}
\hline Variables & $\begin{array}{l}\text { All patients } \\
(\mathrm{n}=142)\end{array}$ & $\begin{array}{l}\text { Non-severe group } \\
(n=125)\end{array}$ & $\begin{array}{l}\text { Severe group } \\
(n=17)\end{array}$ & $\begin{array}{l}P \\
\text { value }\end{array}$ \\
\hline Age (years) & $49.10 \pm 16.36$ & $48.04 \pm 16.66$ & $56.88 \pm 11.61$ & 0.010 \\
\hline Men (\%) & 55 (38.73) & $47(37.60)$ & $8(47.06)$ & 0.453 \\
\hline Body mass index $\left(\mathrm{kg} / \mathrm{m}^{2}\right)$ & $23.81 \pm 3.80$ & $23.50 \pm 3.42$ & $26.13 \pm 5.47$ & 0.007 \\
\hline \multicolumn{5}{|l|}{ Comorbidities (\%) } \\
\hline Diabetes & $12(8.45)$ & $11(8.80)$ & $1(5.88)$ & $>0.999$ \\
\hline Hypertension & $37(26.06)$ & $28(22.40)$ & $9(52.94)$ & 0.007 \\
\hline Cardiovascular disease & $6(4.23)$ & $4(3.20)$ & $2(11.76)$ & $>0.999$ \\
\hline Hepatic disease & $10(7.04)$ & $6(4.80)$ & $4(23.53)$ & 0.131 \\
\hline Chronic lung disease & $9(6.34)$ & $7(5.60)$ & $2(11.76)$ & 0.654 \\
\hline Cancer & $5(3.52)$ & $4(3.20)$ & $1(5.88)$ & $>0.999$ \\
\hline \multicolumn{5}{|l|}{ Initial symptoms (\%) } \\
\hline Fever & $84(59.15)$ & $70(56.00)$ & $14(82.35)$ & 0.038 \\
\hline Nasal congestion & $6(4.23)$ & $5(4.00)$ & $1(5.88)$ & $>0.999$ \\
\hline Sore throat & $18(12.68)$ & $16(12.80)$ & $2(11.76)$ & $>0.999$ \\
\hline Headache/ Dizziness & $10(7.04)$ & $9(7.20)$ & $1(5.88)$ & $>0.999$ \\
\hline Chill & $17(11.9)$ & $13(10.4)$ & $4(23.53)$ & 0.243 \\
\hline Dry mouth & $1(0.70)$ & $0(0.00)$ & $1(5.88)$ & 0.120 \\
\hline Fatigue & $27(19.01)$ & $24(19.20)$ & $3(17.65)$ & $>0.999$ \\
\hline Nausea & $3(2.11)$ & $2(1.60)$ & $1(5.88)$ & 0.320 \\
\hline Myalgia & $10(7.04)$ & $9(7.20)$ & $1(5.88)$ & $>0.999$ \\
\hline Chest distress & $6(4.23)$ & $4(3.20)$ & $2(11.76)$ & 0.315 \\
\hline Cough & $61(42.96)$ & $51(40.80)$ & $10(58.82)$ & 0.159 \\
\hline Expectoration & $32(22.54)$ & $27(21.60)$ & $5(29.41)$ & 0.670 \\
\hline Diarrhea & $5(3.52)$ & $5(4.00)$ & $0(0.00)$ & $>0.999$ \\
\hline Anosmia & $2(1.41)$ & $2(1.60)$ & $0(0.00)$ & $>0.999$ \\
\hline No obvious symptoms & 18 (12.68) & $18(14.40)$ & $0(0.00)$ & 0.199 \\
\hline
\end{tabular}




\begin{tabular}{|c|c|c|c|c|}
\hline Gamma globulin & 88 (61.97) & $78(62.40)$ & $10(58.82)$ & 0.776 \\
\hline Glucocorticoids & $23(16.20)$ & $9(7.20)$ & $14(82.35)$ & $<0.001$ \\
\hline Antibiotics & $52(36.62)$ & $40(32.00)$ & $12(70.59)$ & 0.002 \\
\hline Antivirals & $142(100)$ & $125(100.00)$ & $17(100.00)$ & $>0.999$ \\
\hline Oxygen inhalation & $53(37.32)$ & $36(28.80)$ & $17(100.00)$ & $<0.001$ \\
\hline $\begin{array}{l}\text { Invasive mechanical } \\
\text { ventilation }\end{array}$ & $2(1.41)$ & $0(0.00)$ & $2(11.76)$ & 0.014 \\
\hline $\begin{array}{l}\text { Intensive care unit } \\
\text { admission }\end{array}$ & $3(2.11)$ & $0(0.00)$ & $3(17.65)$ & 0.001 \\
\hline ECMO & $1(0.70)$ & $0(0.00)$ & $1(5.88)$ & 0.120 \\
\hline \multicolumn{5}{|c|}{ Data are presented as mean \pm standard deviation or $\mathrm{n}(\%)$. } \\
\hline \multicolumn{5}{|c|}{$P$ value indicated the comparison between the non-severe group and severe group. } \\
\hline \multicolumn{5}{|c|}{ COVID-19: coronavirus disease 2019, ECMO: extracorporeal membrane oxygenation. } \\
\hline
\end{tabular}

\section{Baseline laboratory parameters}

The baseline laboratory parameters were obtained within 5 days of admission. Compared with those in the non-severe group, patients in the severe group had a higher level of neutrophil\%, fibrinogen, activated partial thromboplastin time (aPTT), C-reactive protein (CRP), interleukin-6 (IL-6), interleukin-10 (IL-10), interferon-y (INF-y), aspartate aminotransferase (AST) and lactic dehydrogenase (LDH), and a lower level of lymphocyte\%, lymphocyte count, platelet count, total cholesterol, high-density lipoprotein cholesterol (HDL-C), low-density lipoprotein cholesterol (LDL-C), apolipoprotein A1 (ApoA1) and albumin (ALB). There were no significant differences in white blood cell (WBC) count, neutrophil count, red blood cell (RBC) count, hemoglobin, D-dimer, prothrombin time (PT), erythrocyte sedimentation rate (ESR), interleukin-2 (IL2), interleukin-4 (IL-4), tumor necrosis factor-a (TNF-a), triglyceride (TG), apolipoprotein B (ApoB), lipoprotein (a), total bilirubin (TBil), Direct bilirubin (DBIL), alanine aminotransferase (ALT), blood urea nitrogen (BUN), blood uric acid (BUA) and serum creatinine ( $\mathrm{Scr}$ ) between the two groups (Table 2). 
Table 2

Baseline laboratory parameters of patients with confirmed COVID-19

\begin{tabular}{|c|c|c|c|c|}
\hline Variables & $\begin{array}{l}\text { All patients } \\
(\mathrm{n}=142)\end{array}$ & $\begin{array}{l}\text { Non-severe group } \\
(n=125)\end{array}$ & $\begin{array}{l}\text { Severe group } \\
(n=17)\end{array}$ & $\begin{array}{l}P \\
\text { value }\end{array}$ \\
\hline WBC count $\left(\times 10^{9}\right)$ & $5.10(4.20-6.80)$ & $5.10(4.25-6.70)$ & $5.30(4.15-7.35)$ & 0.806 \\
\hline Neutrophil\% (\%) & $\begin{array}{l}66.25(58.33- \\
74.50)\end{array}$ & $\begin{array}{l}65.70(57.70- \\
73.15)\end{array}$ & $\begin{array}{l}73.00(65.15- \\
88.55)\end{array}$ & 0.005 \\
\hline Lymphocyte\% (\%) & $\begin{array}{l}24.45(18.50- \\
32.65)\end{array}$ & $\begin{array}{l}25.80(19.05- \\
33.15)\end{array}$ & $\begin{array}{l}19.20(8.55- \\
22.40)\end{array}$ & 0.004 \\
\hline Neutrophil count $\left(\times 10^{9}\right)$ & $3.31(2.48-4.39)$ & $3.27(2.48-4.30)$ & $3.72(2.38-6.22)$ & 0.295 \\
\hline Lymphocyte count $\left(\times 10^{9}\right)$ & $1.23(0.87-1.61)$ & $1.30(0.91-1.66)$ & $0.74(0.48-1.16)$ & 0.001 \\
\hline Platelet count $\left(\times 10^{9}\right)$ & $\begin{array}{l}205.50(155.75- \\
252.25)\end{array}$ & $\begin{array}{l}212.00(165.00- \\
256.00)\end{array}$ & $\begin{array}{l}152.00(120.50- \\
205.00)\end{array}$ & 0.004 \\
\hline RBC count $\left(\times 10^{12}\right)$ & $4.48(4.18-4.93)$ & $4.50(4.22-4.90)$ & $4.32(3.93-5.17)$ & 0.660 \\
\hline Hemoglobin (g/L) & $\begin{array}{l}135.50(125.00- \\
143.25)\end{array}$ & $\begin{array}{l}136.00(125.50- \\
143.00)\end{array}$ & $\begin{array}{l}131.00(121.00- \\
151.00)\end{array}$ & 0.875 \\
\hline D-dimer (ng/ml) * & $\begin{array}{l}100.00(75.00- \\
163.22)\end{array}$ & $\begin{array}{l}100.00(75.00- \\
159.00)\end{array}$ & $\begin{array}{l}158.00(73.00- \\
245.00)\end{array}$ & 0.247 \\
\hline Fibrinogen (mg/dl) & $\begin{array}{l}430.50(370.75- \\
561.00)\end{array}$ & $\begin{array}{l}429.00(362.00- \\
538.50)\end{array}$ & $\begin{array}{l}574.30(406.30- \\
662.00)\end{array}$ & 0.012 \\
\hline Prothrombin time (s) & $\begin{array}{l}12.50(11.50- \\
12.70)\end{array}$ & $\begin{array}{l}12.00(11.50- \\
12.60)\end{array}$ & $\begin{array}{l}12.70(11.55- \\
13.50)\end{array}$ & 0.121 \\
\hline $\begin{array}{l}\text { Activated partial } \\
\text { thromboplastin time (s) }\end{array}$ & $\begin{array}{l}32.45(30.45- \\
35.80)\end{array}$ & $\begin{array}{l}32.30(30.30- \\
35.40)\end{array}$ & $\begin{array}{l}34.80(32.05- \\
41.00)\end{array}$ & 0.048 \\
\hline $\begin{array}{l}\text { Erythrocyte sedimentation } \\
\text { rate }(\mathrm{mm} / \mathrm{h}) \#\end{array}$ & $\begin{array}{l}68.00(41.00- \\
96.00)\end{array}$ & $\begin{array}{l}68.00(38.00- \\
93.25)\end{array}$ & $\begin{array}{l}82.00(57.50- \\
104.50)\end{array}$ & 0.152 \\
\hline C-reactive protein (mg/L) & $\begin{array}{l}8.20(1.64- \\
28.82)\end{array}$ & $4.95(1.26-25.41)$ & $\begin{array}{l}43.95(15.36- \\
71.79)\end{array}$ & $<0.001$ \\
\hline Interleukin-2 (pg/ml) & $0.90(0.56-1.47)$ & $0.90(0.56-1.48)$ & $0.91(0.49-1.51)$ & 0.725 \\
\hline Interleukin-4 (pg/ml) & $1.85(1.17-2.50)$ & $1.85(1.17-2.50)$ & $1.99(1.06-2.63)$ & 0.688 \\
\hline Interleukin-6 (pg/ml) & $\begin{array}{l}3.79(1.87- \\
11.66)\end{array}$ & $3.66(1.84-8.57)$ & $\begin{array}{l}24.11(11.45- \\
51.38)\end{array}$ & $<0.001$ \\
\hline Interleukin-10 (pg/ml) & $3.00(2.02-4.60)$ & $2.98(1.91-4.39)$ & $6.39(2.89-9.55)$ & 0.001 \\
\hline Interferon-y (pg/ml) & $1.19(0.87-1.62)$ & $1.16(0.84-1.51)$ & $1.96(1.27-2.54)$ & $<0.001$ \\
\hline $\begin{array}{l}\text { Tumor necrosis factor- } \mathrm{a} \\
(\mathrm{pg} / \mathrm{ml})\end{array}$ & $1.34(0.98-1.69)$ & $1.34(0.97-1.69)$ & $1.48(1.17-1.73)$ & 0.377 \\
\hline
\end{tabular}




\begin{tabular}{|c|c|c|c|c|}
\hline Total cholesterol (mmol/L) & $4.02(3.57-4.55)$ & $4.08(3.69-4.63)$ & $3.58(3.06-3.85)$ & 0.003 \\
\hline Triglyceride (mmol/L) & $1.42(0.95-2.00)$ & $1.44(0.98-2.00)$ & $1.22(0.83-2.29)$ & 0.540 \\
\hline $\mathrm{HDL}-\mathrm{C}(\mathrm{mmol} / \mathrm{L})$ & $1.08(0.90-1.27)$ & $1.09(0.91-1.29)$ & $0.93(0.82-1.00)$ & 0.020 \\
\hline LDL-C (mmol/L) & $2.50(2.15-2.81)$ & $2.54(2.18-2.85)$ & $2.21(1.93-2.49)$ & 0.024 \\
\hline Apolipoprotein A1 (g/L) & $1.20(1.09-1.31)$ & $1.22(1.12-1.34)$ & $0.98(0.89-1.08)$ & $<0.001$ \\
\hline Apolipoprotein B (g/L) & $0.81(0.70-0.93)$ & $0.81(0.71-0.94)$ & $0.76(0.66-0.86)$ & 0.223 \\
\hline Lipoprotein (a) (mg/L) & $\begin{array}{l}89.45(47.20- \\
147.27)\end{array}$ & $\begin{array}{l}87.15(47.75- \\
161.15)\end{array}$ & $\begin{array}{l}94.45(36.55- \\
135.40)\end{array}$ & 0.794 \\
\hline Albumin (g/L) & $\begin{array}{l}41.45(38.13- \\
44.85)\end{array}$ & $\begin{array}{l}41.90(38.85- \\
45.20)\end{array}$ & $\begin{array}{l}37.30(32.10- \\
41.25)\end{array}$ & $<0.001$ \\
\hline Total bilirubin $(\mu \mathrm{mol} / \mathrm{L})$ & $\begin{array}{l}9.20(6.70- \\
13.65)\end{array}$ & $9.10(6.70-13.60)$ & $\begin{array}{l}11.60(7.30- \\
14.35)\end{array}$ & 0.321 \\
\hline Direct bilirubin $(\mu \mathrm{mol} / \mathrm{L})$ & $3.30(2.40-4.30)$ & $3.20(2.35-4.15)$ & $3.80(3.15-6.15)$ & 0.069 \\
\hline $\begin{array}{l}\text { Aspartate } \\
\text { aminotransferase (IU/L) }\end{array}$ & $\begin{array}{l}23.00(17.00- \\
29.00)\end{array}$ & $\begin{array}{l}22.00(17.00- \\
28.00)\end{array}$ & $\begin{array}{l}28.00(19.50- \\
40.50)\end{array}$ & 0.036 \\
\hline $\begin{array}{l}\text { Alanine aminotransferase } \\
(\mathrm{IU} / \mathrm{L})\end{array}$ & $\begin{array}{l}21.00(14.00- \\
31.00)\end{array}$ & $\begin{array}{l}20.00(14.00- \\
31.00)\end{array}$ & $\begin{array}{l}26.00(18.00- \\
41.50)\end{array}$ & 0.089 \\
\hline Lactic dehydrogenase (IU/L) & $\begin{array}{l}216.00(175.00- \\
248.25)\end{array}$ & $\begin{array}{l}212.00(173.50- \\
239.50)\end{array}$ & $\begin{array}{l}245.00(209.50- \\
350.00)\end{array}$ & 0.006 \\
\hline $\begin{array}{l}\text { Blood urea nitrogen } \\
(\mathrm{mmol} / \mathrm{L})\end{array}$ & $4.23(3.33-5.04)$ & $4.22(3.29-5.02)$ & $4.58(3.62-5.35)$ & 0.259 \\
\hline Blood uric acid $(\mu \mathrm{mol} / \mathrm{L})$ & $\begin{array}{l}267.75(215.82- \\
346.52)\end{array}$ & $\begin{array}{l}276.50(220.35- \\
344.95)\end{array}$ & $\begin{array}{l}253.80(193.40- \\
379.40)\end{array}$ & 0.572 \\
\hline Serum creatinine $(\mu \mathrm{mol} / \mathrm{L})$ & $\begin{array}{l}57.35(48.45- \\
70.60)\end{array}$ & $\begin{array}{l}57.30(48.40- \\
70.55)\end{array}$ & $\begin{array}{l}64.40(47.60- \\
82.35)\end{array}$ & 0.483 \\
\hline
\end{tabular}

Data are presented as medians and inter-quartile ranges.

$P$ value indicated the comparison between the non-severe group and severe group.

* 107 cases in the non-severe group and 11 cases in the severe group tested D-dimer.

\# 98 cases in the non-severe group and 13 cases the non-severe group tested erythrocyte sedimentation rate.

COVID-19: coronavirus disease 2019, WBC: white blood cell, RBC: red blood cell, HDL-C: high-density lipoprotein cholesterol, LDL-C: low-density lipoprotein cholesterol.

\section{Risk factors of the severity of COVID-19}


The univariate logistic analysis showed that age, BMI, hypertension, neutrophil\%, lymphocyte\%, lymphocyte count, platelet count, fibrinogen, aPTT, CRP, IL-6, IL-10, HDL-C, ApoA1, ALB, AST and LDH were associated with the severity of COVID-19. However, only IL-6 (odds ratio [OR]: 1.097, 95\% confidence interval [Cl]: 1.034-1.165, $P=0.002$ ) and ApoA1 (OR: 0.865, 95\% Cl: 0.800-0.935, $P<0.001$ ) were recognized as independent risk factors by multivariate logistic analysis (Table 3 ). 
Table 3

Logistic regression of risk factors for the severity of COVID-19

\begin{tabular}{|c|c|c|c|c|c|c|}
\hline \multirow[t]{2}{*}{ Variables } & \multicolumn{3}{|c|}{ Univariate analysis } & \multicolumn{3}{|c|}{ Multivariate analysis } \\
\hline & OR & $95 \% \mathrm{Cl}$ & $\begin{array}{l}P \\
\text { value }\end{array}$ & OR & $95 \% \mathrm{Cl}$ & $\begin{array}{l}P \\
\text { value }\end{array}$ \\
\hline Age (years) & 10.038 & $\begin{array}{l}1.002- \\
1.075\end{array}$ & 0.041 & & & \\
\hline Body mass index $\left(\mathrm{kg} / \mathrm{m}^{2}\right)$ & 1.186 & $\begin{array}{l}1.041- \\
1.351\end{array}$ & 0.010 & & & \\
\hline Hypertension & 3.897 & $\begin{array}{l}1.376- \\
11.033\end{array}$ & 0.010 & & & \\
\hline Neutrophil\% (\%) & 1.071 & $\begin{array}{l}1.022- \\
1.123\end{array}$ & 0.004 & & & \\
\hline Lymphocyte\% (\%) & 0.924 & $\begin{array}{l}0.872- \\
0.978\end{array}$ & 0.007 & & & \\
\hline Lymphocyte count $\left(\times 10^{9}\right)$ & 0.154 & $\begin{array}{l}0.043- \\
0.549\end{array}$ & 0.004 & & & \\
\hline Platelet count $\left(\times 10^{9}\right)$ & 0.988 & $\begin{array}{l}0.979- \\
0.998\end{array}$ & 0.015 & & & \\
\hline Fibrinogen (mg/dl) & 1.005 & $\begin{array}{l}1.001- \\
1.008\end{array}$ & 0.005 & & & \\
\hline $\begin{array}{l}\text { Activated partial thromboplastin } \\
\text { time (s) }\end{array}$ & 1.083 & $\begin{array}{l}0.997- \\
1.176\end{array}$ & 0.060 & & & \\
\hline C-reactive protein (mg/L) & 1.03 & $\begin{array}{l}1.013- \\
1.046\end{array}$ & $<0.001$ & & & \\
\hline Interleukin-6 (pg/ml) & 1.096 & $\begin{array}{l}1.050- \\
1.144\end{array}$ & $<0.001$ & 1.097 & $\begin{array}{l}1.034- \\
1.165\end{array}$ & 0.002 \\
\hline Interleukin-10 (pg/ml) & 1.121 & $\begin{array}{l}1.020- \\
1.231\end{array}$ & 0.017 & & & \\
\hline Interferon-y (pg/ml) & 0.996 & $\begin{array}{l}0.955- \\
1.038\end{array}$ & 0.840 & & & \\
\hline Total cholesterol (mmol/L) & 0.558 & $\begin{array}{l}0.288- \\
1.081\end{array}$ & 0.084 & & & \\
\hline HDL-C (mmol/L) & 0.088 & $\begin{array}{l}0.008- \\
0.931\end{array}$ & 0.043 & & & \\
\hline LDL-C (mmol/L) & 0.591 & $\begin{array}{l}0.258- \\
1.352\end{array}$ & 0.213 & & & \\
\hline Apolipoprotein A1 (mg/dl) & 0.885 & $\begin{array}{l}0.839- \\
0.934\end{array}$ & $<0.001$ & 0.865 & $\begin{array}{l}0.800- \\
0.935\end{array}$ & $<0.001$ \\
\hline
\end{tabular}




$\begin{array}{llll}\text { Albumin (g/L) } & 0.791 & \begin{array}{l}0.700- \\ 0.893\end{array} & <0.001 \\ & & & \\ \text { Aspartate aminotransferase (IU/L) } & 1.036 & \begin{array}{l}1.006- \\ 1.066\end{array} & 0.016 \\ & & & \\ \text { Lactic dehydrogenase (IU/L) } & 1.009 & \begin{array}{l}1.003- \\ 1.016\end{array} & 0.003\end{array}$

COVID-19: coronavirus disease 2019, OR: odd ratio, Cl: confidence interval, HDL-C: high-density lipoprotein cholesterol, LDL-C: low-density lipoprotein cholesterol

A risk model was established upon ApoA1 and IL-6. For predicting the severity of COVID-19, the area under ROC curves (AUROCs) for ApoA1, IL-6 and risk model were 0.896 (95\% Cl: 0.834-0.941), 0.855 (95\% Cl: $0.786-0.908)$ and $0.977(95 \% \mathrm{Cl}: 0.932-0.995)$, respectively (Figure 1, Table 4).

Table 4

Predictive performance of apolipoprotein A1, interleukin- 6 and risk model for the severity of COVID-19

\begin{tabular}{|lllllll}
\hline Variables & $\begin{array}{l}\text { AUROC } \\
(95 \% \mathrm{Cl})\end{array}$ & $\begin{array}{l}\text { Cut-off } \\
\text { value }\end{array}$ & $\begin{array}{l}\text { Sensitivity } \\
\%\end{array}$ & $\begin{array}{l}\text { Specificity } \\
\%\end{array}$ & $\begin{array}{l}\text { PPV \% } \\
(95 \% \mathrm{Cl})\end{array}$ & $\begin{array}{l}\text { NPV \% } \\
(95 \% \mathrm{Cl})\end{array}$ \\
\hline $\begin{array}{l}\text { Apolipoprotein } \\
\text { A1 }\end{array}$ & 0.896 & $1.09 \mathrm{~g} / \mathrm{L}$ & 94.12 & 80.80 & 40.00 & 99.00 \\
& $\begin{array}{l}(0.834- \\
0.941)\end{array}$ & & $\begin{array}{l}(71.20- \\
99.00)\end{array}$ & $\begin{array}{l}(72.80- \\
87.30)\end{array}$ & $\begin{array}{l}(24.90- \\
56.70)\end{array}$ & $\begin{array}{l}(94.60- \\
\text { Interleukin-6 }\end{array}$ \\
& 0.855 & 9.65 & 88.24 & 77.60 & 34.90 & 98.00 \\
& $(0.786-$ & $\mathrm{pg} / \mathrm{ml}$ & $(63.50-$ & $(69.30-$ & $(21.00-$ & $(92.90-$ \\
& $0.908)$ & & $98.20)$ & $84.60)$ & $50.90)$ & $99.70)$ \\
\hline Risk model & 0.977 & $/$ & 100.00 & 88.89 & 58.60 & 100.00 \\
& $(0.932-$ & & $(80.30-$ & $(81.40-$ & $(38.90-$ & $(96.20-$ \\
& $0.995)$ & & $100.00)$ & $94.10)$ & $76.50)$ & $100.00)$ \\
\hline
\end{tabular}

COVID-19: coronavirus disease 2019, PPV: Positive predictive value, NPV: Negative predictive value

Correlation analyses showed that ApoA1 positively correlated with lymphocyte count $(\mathrm{r}=0.257, P=0.002)$, HDL-C ( $\mathrm{r}=0.681, P<0.001)$ and ALB $(\mathrm{r}=0.412, P<0.001)$, while negatively correlated with fibrinogen $(\mathrm{r}=0.227, P=0.001), \mathrm{CRP}(\mathrm{r}=-0.337, P<0.001)$ and AST $(\mathrm{r}=-0.240, P=0.004)$. No correlations were found between ApoA1 and age, hypertension, neutrophil count, platelet count, IL-6, IL-10 and ALT (Figure 2).

\section{Dynamic change of ApoA1}

The baseline ApoA1 in the severe group was significantly lower than that in the non-severe group $(P<0.001)$. While, the ApoA1 level in the severe group elevated during the treatment $(P<0.001)$, but it was 
not found in the non-severe group $(P=0.223)$. In the recovery stage of COVID-19, no significant difference was found between the two groups $(P=0.560)$ (Figure 3$)$.

\section{Discussion}

In this study, we found that the blood lipid in the severe patients with COVID-19 was differ from that in the non-severe patients, which showed baseline total cholesterol, HDL-C, LDL-C ApoA1 in the severe group were much lower than those in the non-severe group, while TG, ApoB and Lipoprotein (a) had no significant differences between the two groups. Additionally, ApoA1 was recognized as an independent risk factor for the severity of disease revealed by multivariate logistic analysis. Furthermore, ApoA1 had the highest AUROC among all the single markers for the severity of COVID-19, and the combination of ApoA1 and IL-6 got a higher AUROC. On the other hand, the dynamic raise of ApoA1 in the severe patients was parallel to the improvement of the disease.

Previous studies have found that lipid metabolism impairment may involve in the pathogenesis of sepsis secondly to pneumonia and influenza [10-12]. Similarly, recent studies observed the dyslipidemia in the patients infected with SARS-CoV-2 by MS analysis, especially in the severe cases[7-9], which reminded us that blood lipid might serve as a maker for the severity of COVID-19. While among the altered lipids, ApoA1 was very obviously decreased.

ApoA1, a major protein component of HDL complex, is known to involved in the "reverse cholesterol transport" by transporting cholesterol excess from peripheral cells back to the liver for excretion. Apart from it, ApoA1 also takes a part in the anti-inflammatory [13]. In acute inflammatory disease, serum amyloid A (SAA), an acute phase protein, displace ApoA1 from the HDL complex, then the free ApoA1 is easily eliminated by the kidney, resulting in a low level in the peripheral blood [14]. On the other hand, liver is susceptible to be attacked by SARS-CoV-2, especially in the severe cases [15], therefore, a decrease synthesis by the injured liver may also played a role.

Some previous studies have revealed that serum ApoA1 is associated with the outcome of patients with sepsis, acute respiratory distress syndrome induced by pneumonia, and critical ill [16-19]. A recent research observed that low level ApoA1 was associated with the severity of COVID-19, with an AUROC of 0.728 in predicting its severity [20]. Our study confirmed its role in distinguishing the severe cases, however, the predictability of ApoA1 in our study was even higher than the former, with an AUROC as high as 0.896 (95\% Cl:0.834-0.941), and recognized as a risk factor for the severity of COVID-19. The discrimination may be related to the different patients included. Our study group enrolled all clinical types which consist of mild, moderate, severe and critical, while the former excluded the critical type. Moreover, although the sample size in our study was larger, the patients in the severe group $(17,11.97 \%)$ was smaller than the former $(25,25.77 \%)$. Additionally, the former study performed in Wuhan, the most affected area by the COVID-19 outbreak in China, therefore, the laboratory examination may be delayed. Thus, the different time of baseline detection may also take a part. 
As well known, IL-6 plays a key role in the development of COVID-19, and its predictive value have been revealed by us previously and others $[5,21]$. In this study, IL -6 and ApoA1 were observed to be independent risk factors. Additionally, there was no significant correlation between IL-6 and ApoA1. Based on their complementarity, the risk model established by these two markers exhibited the highest predictability with AUROC of 0.977 (95\% Cl: 0.932-0.995).

ApoA1, and its mimetic peptide $D$-amino acids (D-4F) have showed their therapeutic potential in treating cancer, influenza, sepsis and a variety of lung diseases, such as acute respiratory distress syndrome (ARDS), mainly due to its anti-inflammatory, anti-oxidant and anti-apoptotic properties [13,22-25]. In addition, it was noteworthy that ApoA1 could inhibit the release of IL-6 and reduce the activation of macrophage. IL -6 was the main participant in cytokine storm, and macrophage was the primary source of IL-6. Therefore, ApoA1 may have therapeutic potential in treating patients with COVID-19.

There were some limitations in this study. First, it was a single center retrospective study. Second, the sample size was relatively small, especially of the severe cases. Third, it was not validated by internal and external cohorts. Therefore, a prospective study with a large sample size is warranted.

\section{Conclusion}

In conclusion, our study shed light on a different blood lipid profile in the severe patients with COVID-19 from non-severe patients, which manifest as low level of total cholesterol, HDL-C, LDL-C and ApoA1 in the severe group. ApoA1 is the best predictor for the severity of COVID-19 among all the single markers in this study, and the combination of ApoA1 and IL-6 enhanced the predictability. Furthermore, ApoA1 could serve as a tool to monitor the course of COVID-19.

\section{Abbreviations}

COVID-19: coronavirus disease 2019; HDL-C: high-density lipoprotein cholesterol; LDL-C: low-density lipoprotein cholesterol; ApoA1: apolipoprotein A1; IL-6: interleukin-6; AUROC: highest area under the receiver operator characteristic curve; SARS-CoV-2: severe acute respiratory syndrome coronavirus 2; EMR: electronic medical record.

\section{Declarations}

\section{Acknowledgements}

We thank all the medical staff of HwaMei Hospital, University of Chinese Academy of Sciences for their hard work and enormous contributions during the outbreak of COVID-19.

\section{Authors' Contribution}


Yayun Yang and Zhe Zhu analyzed the data and wrote the manuscript. Linyan Fan collected clinical data and follow up. Shuyuan Ye and Kehong Lou collected the library data. Xin Hua, Zuoan Huang and Qiaoyun Shi performed laboratory analysis. Guosheng Gao designed the study and revised the manuscript.

\section{Funding}

This study was supported by the COVID-19 Platform Program of Hwa Mei Hospital, University of Chinese Academy of Sciences (2020HMZD21, 2020HMZD27).

\section{Availability of data and materials}

The datasets used and analyzed during the current study are available from the corresponding author on reasonable request.

\section{Ethics approval and consent to participate}

This study was approved by the Ethics Committee of Hwa Mei Hospital, University of Chinese Academy of Sciences (Certificate no. PJ-NBEY-KY-2020-061-01). Written informed consent was obtained from all participants.

\section{Consent for publication}

Written informed consent was obtained from all participants.

\section{Competing interests}

The authors declare no conflict of interest.

\section{References}

1. Epidemiology Working Group for Ncip Epidemic Response CCfDC, Prevention: The epidemiological characteristics of an outbreak of 2019 novel coronavirus diseases (COVID-19) in China. Zhonghua Liu Xing Bing Xue Za Zhi. 2020; 41:145-51.

2. Yang $X, Y u$ Y, Xu J, Shu H, Xia J, Liu H, Wu Y, Zhang L, Yu Z, Fang M, et al: Clinical course and outcomes of critically ill patients with SARS-CoV-2 pneumonia in Wuhan, China: a single-centered, retrospective, observational study. Lancet Respir Med. 2020; 8:475-81. 
3. Ye Q, Wang B, Mao J: The pathogenesis and treatment of the 'Cytokine Storm' in COVID-19. J Infect. 2020; doi: 10.1016/j.jinf.2020.03.037. [Epub ahead of print]

4. Wang D, Hu B, Hu C, Zhu F, Liu X, Zhang J, Wang B, Xiang H, Cheng Z, Xiong Y, et al: Clinical Characteristics of 138 Hospitalized Patients With 2019 Novel Coronavirus-Infected Pneumonia in Wuhan, China. JAMA. 2020; doi:10.1001/jama.2020.1585. [Epub ahead of print]

5. Zhu Z, Cai T, Fan L, Lou K, Hua X, Huang Z, Gao G: Clinical value of immune-inflammatory parameters to assess the severity of coronavirus disease 2019. Int J Infect Dis. 2020; doi: 10.1016/j.ijid.2020.04.041. [Epub ahead of print]

6. Liu J, Li S, Liu J, Liang B, Wang X, Wang H, Li W, Tong Q, Yi J, Zhao L, et al: Longitudinal characteristics of lymphocyte responses and cytokine profiles in the peripheral blood of SARS-CoV-2 infected patients. EBioMedicine. 2020; 55:102763.

7. Wu D, Shu T, Yang X, Song J-X, Zhang M, Yao C, Liu W, Huang M, Yu Y, Yang Q, et al: Plasma Metabolomic and Lipidomic Alterations Associated with COVID-19. National Science Review. 2020; https://doi.org/10.1093/nsr/nwaa086.

8. Messner CB, Demichev V, Wendisch D, Michalick L, White M, Freiwald A, Textoris-Taube K, Vernardis SI, Egger A-S, Kreidl M, et al: Clinical classifiers of COVID-19 infection from novel ultra-highthroughput proteomics. medRxiv. 2020; doi: https://doi.org/10.1101/2020.04.27.20081810.

9. Shen B, Yi X, Sun Y, Bi X, Du J, Zhang C, Quan S, Zhang F, Sun R, Qian L, et al: Proteomic and Metabolomic Characterization of COVID-19 Patient Sera. medRxiv. 2020; doi: https://doi.org/10.1101/2020.04.07.20054585.

10. Sharma NK, Ferreira BL, Tashima AK, Brunialti MKC, Torquato RJS, Bafi A, Assuncao M, Azevedo LCP, Salomao R: Lipid metabolism impairment in patients with sepsis secondary to hospital acquired pneumonia, a proteomic analysis. Clin Proteomics. 2019; 16:29.

11. Sharma NK, Tashima AK, Brunialti MKC, Ferreira ER, Torquato RJS, Mortara RA, Machado FR, Assuncao M, Rigato O, Salomao R: Proteomic study revealed cellular assembly and lipid metabolism dysregulation in sepsis secondary to community-acquired pneumonia. Sci Rep. 2017; 7:15606.

12. Tisoncik-Go J, Gasper DJ, Kyle JE, Eisfeld AJ, Selinger C, Hatta M, Morrison J, Korth MJ, Zink EM, Kim YM, et al: Integrated Omics Analysis of Pathogenic Host Responses during Pandemic H1N1 Influenza Virus Infection: The Crucial Role of Lipid Metabolism. Cell Host Microbe. 2016; 19:254-66.

13. Peng M, Zhang Q, Cheng Y, Fu S, Yang H, Guo X, Zhang J, Wang L, Zhang L, Xue Z, et al: Apolipoprotein A-I mimetic peptide 4F suppresses tumor-associated macrophages and pancreatic cancer progression. Oncotarget. 2017; 8:99693-706.

14. Tanaka S, Couret D, Tran-Dinh A, Duranteau J, Montravers P, Schwendeman A, Meilhac O: Highdensity lipoproteins during sepsis: from bench to bedside. Crit Care. 2020; 24:134.

15. Xu L, Liu J, Lu M, Yang D, Zheng X: Liver injury during highly pathogenic human coronavirus infections. Liver Int. 2020; 40:998-1004.

16. Chien JY, Jerng JS, Yu CJ, Yang PC: Low serum level of high-density lipoprotein cholesterol is a poor prognostic factor for severe sepsis. Crit Care Med. 2005; 33:1688-93. 
17. Barlage S, Gnewuch C, Liebisch G, Wolf Z, Audebert FX, Gluck T, Frohlich D, Kramer BK, Rothe G, Schmitz G: Changes in HDL-associated apolipoproteins relate to mortality in human sepsis and correlate to monocyte and platelet activation. Intensive Care Med. 2009; 35:1877-85.

18. Ren S, Chen X, Jiang L, Zhu B, Jiang Q, Xi X: Deleted in malignant brain tumors 1 protein is a potential biomarker of acute respiratory distress syndrome induced by pneumonia. Biochem Biophys Res Commun. 2016; 478:1344-9.

19. Chenaud C, Merlani PG, Roux-Lombard P, Burger D, Harbarth S, Luyasu S, Graf JD, Dayer JM, Ricou B: Low apolipoprotein A-I level at intensive care unit admission and systemic inflammatory response syndrome exacerbation. Crit Care Med. 2004; 32:632-7.

20. Nie S, Zhao X, Zhao K, Zhang Z, Zhang Z, Zhang Z: Metabolic disturbances and inflammatory dysfunction predict severity of coronavirus disease 2019 (COVID-19): a retrospective study. medRxiv. 2020; doi: https://doi.org/10.1101/2020.03.24.20042283.

21. Liu F, Li L, Xu M, Wu J, Luo D, Zhu Y, Li B, Song X, Zhou X: Prognostic value of interleukin-6, C-reactive protein, and procalcitonin in patients with COVID-19. J Clin Virol. 2020; 127:104370.

22. Van Lenten BJ, Wagner AC, Navab M, Anantharamaiah GM, Hui EK, Nayak DP, Fogelman AM: D-4F, an apolipoprotein A-I mimetic peptide, inhibits the inflammatory response induced by influenza $A$ infection of human type II pneumocytes. Circulation. 2004; 110:3252-8.

23. Van Lenten BJ, Wagner AC, Anantharamaiah GM, Garber DW, Fishbein MC, Adhikary L, Nayak DP, Hama S, Navab M, Fogelman AM: Influenza infection promotes macrophage traffic into arteries of mice that is prevented by D-4F, an apolipoprotein A-I mimetic peptide. Circulation. 2002; 106:1127-32.

24. Guo L, Ai J, Zheng Z, Howatt DA, Daugherty A, Huang B, Li XA: High density lipoprotein protects against polymicrobe-induced sepsis in mice. J Biol Chem. 2013; 288:17947-53.

25. Gordon EM, Figueroa DM, Barochia AV, Yao X, Levine SJ: High-density Lipoproteins and Apolipoprotein A-l: Potential New Players in the Prevention and Treatment of Lung Disease. Front Pharmacol. 2016; 7:323.

\section{Figures}




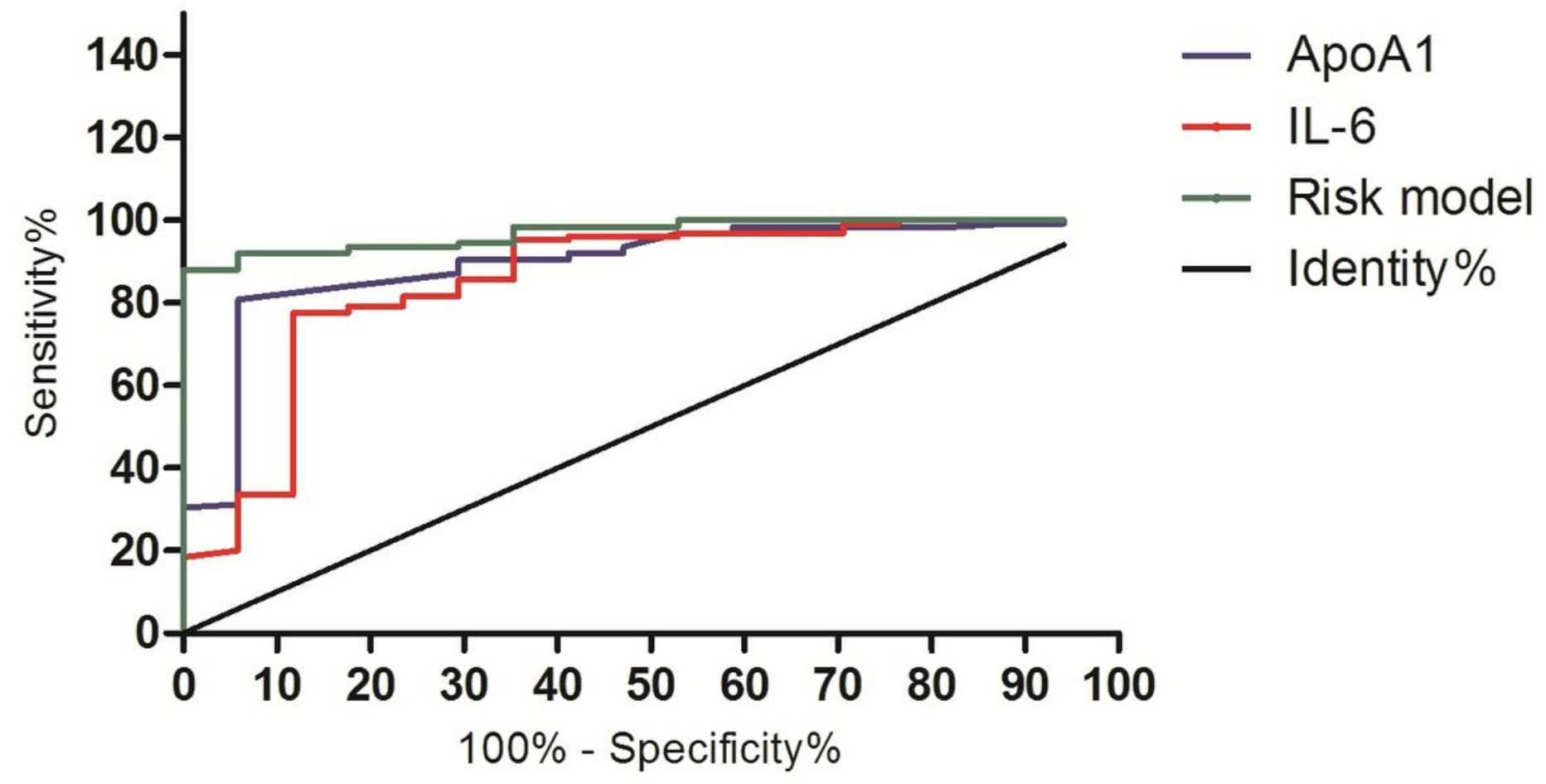

Figure 1

Receiver operator characteristic curves of ApoA1, IL- 6 and risk model for the severity of COVID-19. COVID19: coronavirus disease 2019, ApoA1: apolipoprotein A1, IL-6: interleukin-6 


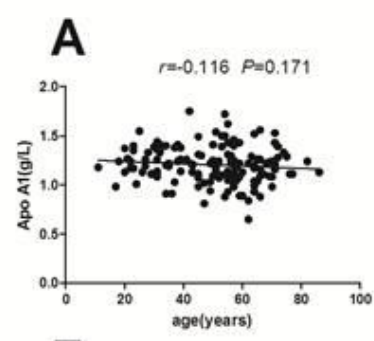

E
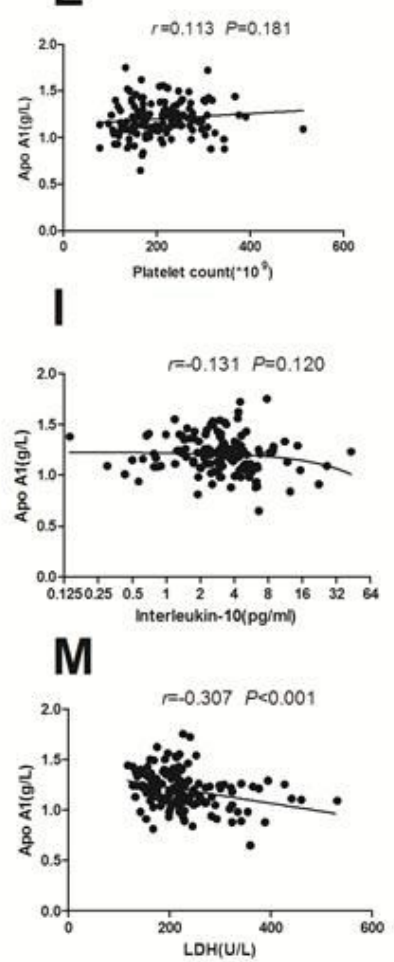
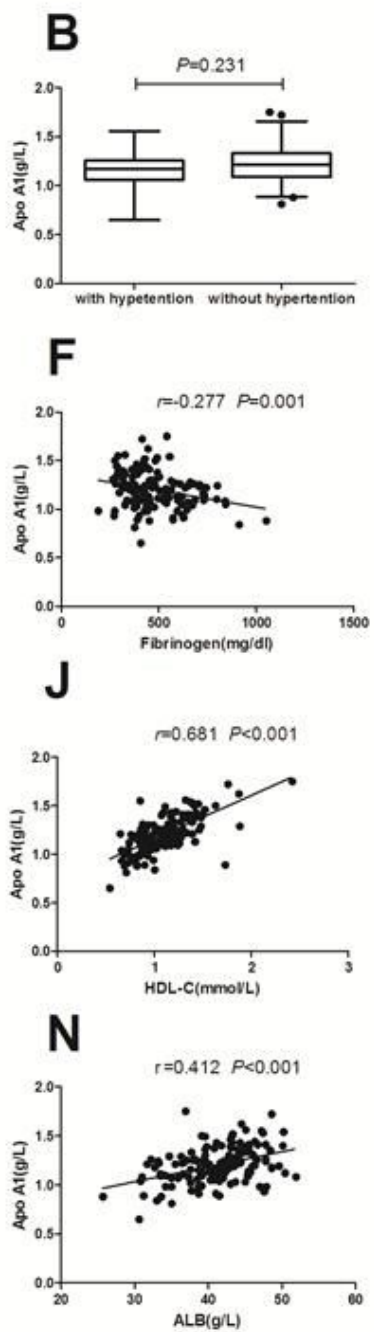

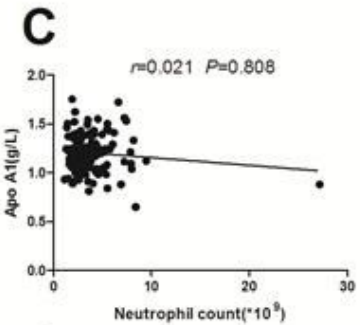

G
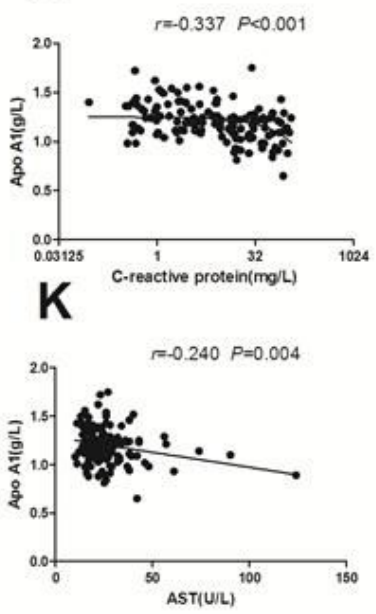

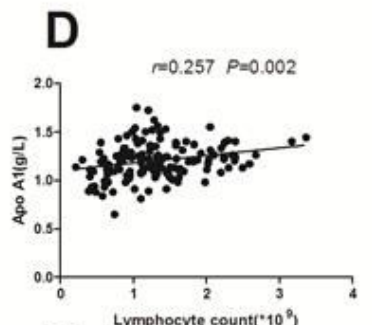

H
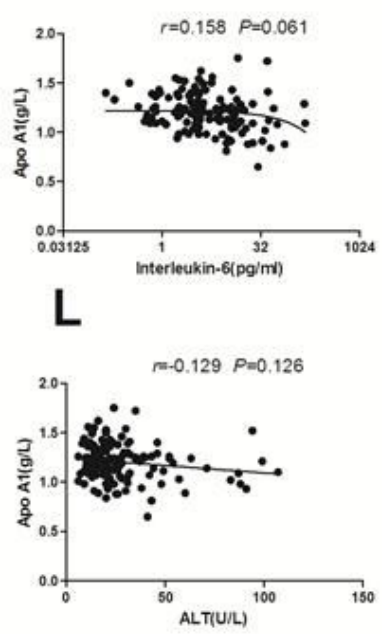

Figure 2

Correlations between ApoA1 and other parameters in patients with COVID-19. COVID-19: coronavirus disease 2019, ApoA1: apolipoprotein A1, HDL-C: high-density lipoprotein cholesterol, AST: aspartate aminotransferase, ALT: alanine aminotransferase, LDH: lactic dehydrogenase, ALB: albumin 

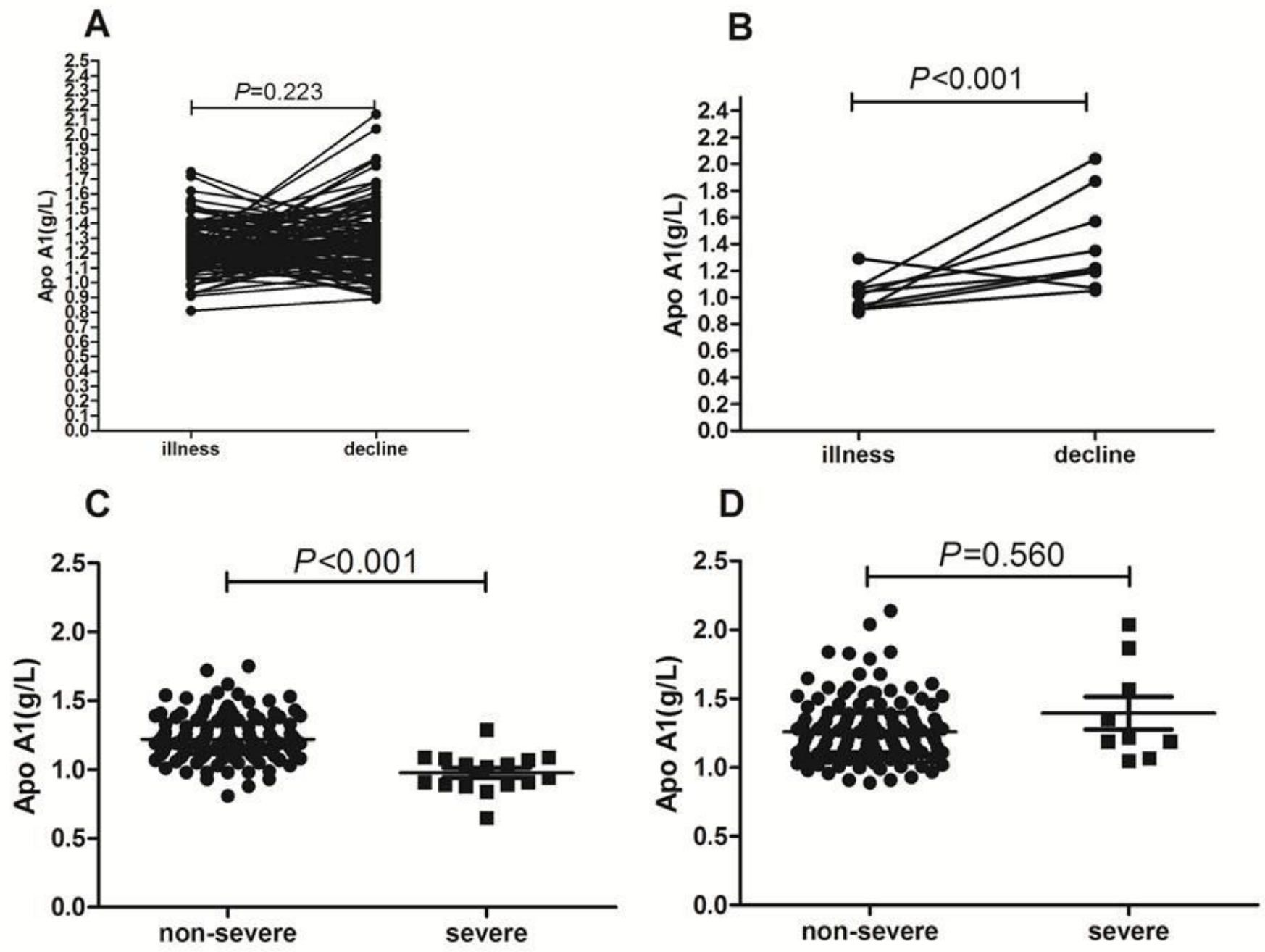

\section{Figure 3}

Dynamic change of ApoA1 in patients with COVID-19. (A). Dynamic change of ApoA1 in the non-severe group; (B). Dynamic change of ApoA1 in the severe group; (C). The comparison of ApoA1 between the non-severe and severe group at baseline. (D) The comparison of ApoA1 between the non-severe and severe group in the recovery stage of disease. COVID-19: coronavirus disease 2019, ApoA1: apolipoprotein A1 